\title{
El alumno CLIL: resultados del impacto de una página web en la adquisición de una segunda lengua
}

\section{The CLIL student: results of the impact of a webpage on Second Language Acquisition}

\author{
DELGADILLO, Mónica†*, RODRÍGUEZ, Norma y TORRES, Sandra \\ Universidad Tecnológica Fidel Velázquez. Departamento de Idiomas/División Académica de Informática.
}

ID $1^{\text {er }}$ Autor: Mónica, Delgadillo / ORC ID: 0000-0003-1279-0495, Researcher ID Thomson: X-2166-2018, CVU CONACYT ID: 313108

ID $1^{\text {er }}$ Coautor: Norma, Rodríguez / ORC ID: 0000-0002-8793-8602, Researcher ID Thomson: X-2200-2018, Open ID: 112329307586159931670

ID $2^{\text {do }}$ Coautor: Sandra, Torres / ORC ID: 0000-0002-9616-8849, Researcher ID Thomson: X-2147-2018

DOI: $10.35429 / \mathrm{JAD} \cdot 2019.7 .3 .22 .25$

Recibido 15 de Enero, 2019; Aceptado 28 de Marzo, 2019

\begin{abstract}
Resumen
La implementación de las Tecnologías de Información y Comunicación (TIC) en el área educativa ha generado toda una revolución en los procesos y métodos de enseñanza existentes, y cada vez son más las plataformas virtuales que median el proceso enseñanza-aprendizaje (Rodríguez, 2010). Dichas herramientas posibilitan de forma rápida y eficiente algunos procesos como la comunicación, un cálculo o programa mediante la automatización. El presente documento aborda los resultados de la investigación realizada para medir el impacto del uso de una página gratuita desarrollada para el aprendizaje del idioma inglés en la Universidad Tecnológica Fidel Velázquez. El estudio fue descriptivo y se emplearon dos grupos para contrastar el comportamiento de ambos al utilizar la página desarrollada por el Cuerpo Académico de Lenguas Extranjeras. Se llevó un registro estadístico y se aplicaron encuestas para conocer opinión de usuarios al emplear dicha aplicación. La finalidad de la página es proporcionar a la comunidad interna y externa contenidos específicos en inglés para enriquecer su vocabulario y reconocer la utilidad de una segunda lengua en su área de desempeño profesional.
\end{abstract}

Bilingüismo, CLIL, Eficacia escolar

\begin{abstract}
The implementation of Information and Communication Technologies (ICT) in the educational area has generated a revolution in the existing teaching processes and methods, and nowadays more virtual platforms help to mediate the teaching-learning process (Rodríguez, 2010). These tools enable quickly and efficiently some processes such as communication, a calculation or program through automation. This document addresses the results of the research conducted to measure the impact of using a free page developed for English language learning at Universidad Tecnológica Fidel Velázquez. The study was descriptive: two groups were used to contrast the behavior when using the page developed by the Academic Body of Foreign Languages. A statistical record was kept and surveys were applied to know users opinion when employing it. The purpose of the page is to provide the internal and external community with specific content in English to strengthen vocabulary and recognize the usefulness of a second language in their area of professional performance.
\end{abstract}

Bilingualism, CLIL, School efectiveness

Citación: DELGADILLO, Mónica, RODRÍGUEZ, Norma y TORRES, Sandra. El alumno CLIL: resultados del impacto de una página web en la adquisición de una segunda lengua. Revista de Arquitectura y Diseño. 2019. 3-7: 22-25.

\footnotetext{
*Correspondencia al Autor (Correo Electrónico: monica.delgadillo@utfv.edu.mx)

$\dagger$ Investigador contribuyendo como primer autor.
} 


\section{Introducción}

CLIL es la abreviatura de Content and Language Integrated Learning. Se refiere básicamente a la enseñanza del contenido de materias curriculares empleando una lengua extranjera. Los alumnos adquieren conocimiento y comprensión de la materia curricular mientras que simultáneamente aprenden y usan el inglés.

El uso de CLIL permite que durante el aprendizaje los alumnos sientan la necesidad de usar correctamente el lenguaje específico: si es de ciencias exactas $o$ bien del área administrativa o social.

Los profesores que estén inmersos en este tipo de programa por su parte deberán tener un manejo adecuado del inglés (nivel B1/B2 por lo menos) y conocer el vocabulario específico que requieren los alumnos para ser competentes en dicha área. Debido a ello los profesores deberán estar recibiendo capacitación continua en el manejo de contenidos en inglés por área específica (CLIL).

En el caso de la Universidad Tecnológica Fidel Velázquez, se implementó el programa bajo el nombre de Programa BIS (Bilingüe, Internacional y Sustentable), el cual tiene el propósito de capacitar a los docentes en el manejo del inglés para impartir su asignatura en idioma inglés. A la par de lo anterior se diseñó un sitio por parte del Cuerpo Académico de Lengua Extranjera con el fin de apoyar a los alumnos y docentes con un espacio que les permita acceder a material complementario por áreas de conocimiento para mayor práctica.

La ventaja del sitio es que está enfocado a los contenidos que los alumnos revisan en clase y que pueden acceder a ellos cuantas veces quieran. El sitio es abierto y debido a falta de recurso se ha hecho con recursos gratuitos existentes en la red, lo cual por otro lado también ha limitado la cantidad de material, sin embargo ha recibido comentarios positivos por parte de docentes y alumnos. En este artículo se presentan los resultados preliminares de dicho sitio. En la Sección 2 se ubica la revisión bibliográfica que realizamos para el planteamiento de la hipótesis. En la Sección 3 se presentan metodología y los datos de la página para probar la hipótesis.
En la Sección 4 se muestran los resultados de las encuestas de satisfacción aplicadas a los alumnos de los grupos piloto. En la sección 5 se ubican las conclusiones y las referencias bibliográficas.

\section{Sección 2}

\section{Revisión bibliográfica}

Se realizó una revisión bibliográfica respecto a lo existente en el tema y se encontró que ya hay una extensa contribución al tema por parte de varios investigadores como Casañ y ríos Márquez que han estudiado el impacto que tiene el uso de CLIL en el aprendizaje de una segunda lengua, además del uso de TI como herramientas en el aprendizaje.

Se plantea que a través del empleo de CLIL se mejorará la adquisición del idioma inglés ya que el alumno estará en contacto con terminología relacionada con su área y por ende será capaz de incrementar su vocabulario, lo cual le permitirá comunicarse eficientemente.

\section{Sección 3}

\section{Metodología}

Se aplicó una metodología de corte exploratorio, se realizó una revisión documental en diversas fuentes para conocer lo existente respecto al tema. Se diseñó el sitio empleando recursos abiertos y se seleccionó el material procedente de fuentes auténticas en algunos casos y en otros de libros de texto: en todos los casos se dan créditos de autoría a la fuente en cuestión. El material que se eligió para el sitio comprende contenidos de materias como Integradora, que es una de las materias que los alumnos emplean para desarrollar proyectos de investigación, y que les permitirá, como lo indica su nombre, integrar lo aprendido en su campo de formación. Para ingresar al sitio requieren teclear únicamente su nombre y en automático los dirige al sito para que realicen las actividades. Se requierió también revisar que modificaciones se deben revisar en el trabajo docente para que incluyan el uso del sitio en sus actividades formativas. Los docentes realmente solo requieren seleccionar el material que desean incluir y que sus alumnos realicen las actividades. 
El Cuerpo Académico de Lenguas Extranjeras revisa el contenido y la pertinencia de éste. En caso de ser necesaria una corrección en cuanto a estructura gramatical, se le hace la observación al docente para que lo corrija y pueda ser cargado en el contenido. El estudio se aplicó en dos grupos: uno que fungió como testigo y otro de prueba. El grupo testigo asistió a clases tradicionales de inglés, sin usar el sitio. En el caso del grupo de prueba, los alumnos realizaron los ejercicios del sitio como parte de su evaluación continua, con el fin de forzarlos a que la usaran. Posteriormente se aplicó un cuestionario en el cual se le preguntó al usuario cual es su opinión acerca del uso de dicho material como apoyo en el aprendizaje del inglés. Se aplicó un examen diagnóstico para conocer el nivel de ambos grupos antes del estudio; y al finalizar el cuatrimestre se aplicó un examen de salida para conocer el grado de avance de ambos.

\section{Sección 4}

\section{Resultados}

De acuerdo a los resultados obtenidos en los cuestionarios de satisfacción, el uso del sitio fue de mucha utilidad para los alumnos en su clase de inglés, ya que mencionan que es más fácil repasar de esa forma el vocabulario de su área.

Los alumnos que emplearon el recurso CLIL obtuvieron una mejor calificación en la materia de inglés en comparación con el grupo testigo, lo cual les produjo un incentivo para seguir participando en el uso del sito para reforzar su vocabulario y las estructuras gramaticales. Los docentes, por su parte indicaron que el participar en dicho proyecto fue demandante y al mismo tiempo gratificante pues aprendieron vocabulario nuevo, aunque requirió un trabajo extra para ellos. Uno de los puntos que más costó trabajo a los docentes fue acostumbrarse al uso de las TI y combinarlas con el empleo de CLIL en sus clases, ya que también fueron evaluados por los docentes, lo cual fue estresante para ellos. Aunque el resultado obtenido es alentador, varios de los profesores tienen problemas para la ortografía de algunos términos de su área y la pronunciación sigue siendo el mayor reto a vencer, pues varios alumnos tienen mejor pronunciación que los docentes, lo cual en algunos casos resultó incomodo para los maestros al verse superados por los alumnos.
Un problema recurrente fue el hecho de que en ocasiones se saturaba la página, a pesar de que eran relativamente pocos los alumnos que la estaban empleando, y el problema será mayor si se hace extensivo el uso a más grupos, por lo que tendemos que revisarla y hacer las adecuaciones pertinentes al respecto.

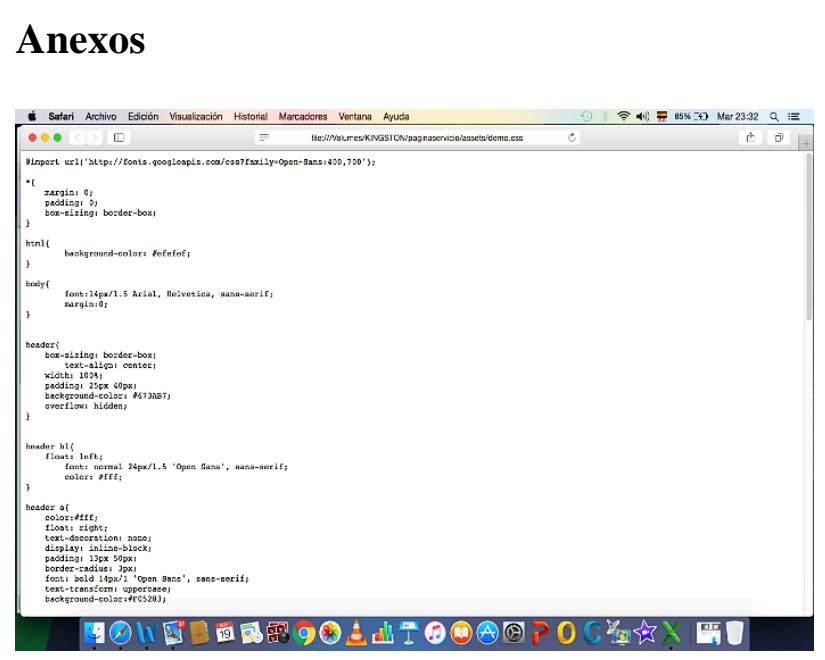

Figura 1 Programación de la página Fuente: elaboración propia

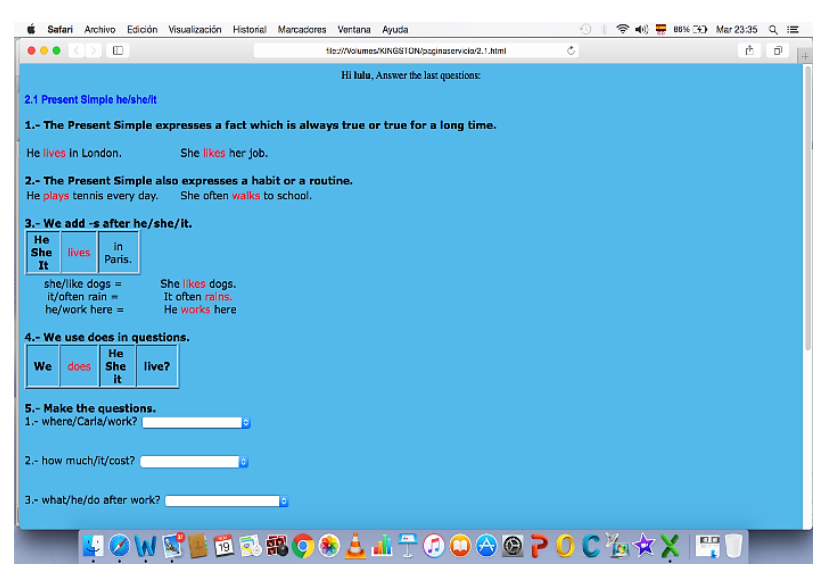

Figura 2 Ejemplo de ejercicios en el sitio Fuente: elaboración propia

\section{Agradecimiento}

Gracias a la Universidad Tecnológica Fidel Velázquez por su apoyo para la realización de este proyecto.

\section{Sección 5}

\section{Conclusiones}

El uso de CLIL en el aprendizaje del idioma inglés proporciona al alumno acceso a mayor cantidad de vocabulario relacionado con su área. 
Al encontrarse con el idioma aplicado en su área de desempeño profesional, el alumno logra encontrar la aplicación del mismo, y por lo tanto el aprendizaje logra insertarse en su Zona de Desempeño Próximo Distal, volviéndose un conocimiento útil para el alumno.

Una de las grandes dificultades radica en convencer al docente del área respectiva para que se capacite en el manejo del idioma inglés, ya que el salario que percibe es el mismo, lo cual le resta atractivo a la impartición de clases empleando CLIL para tal efecto.

Si se pretende incentivar el manejo del inglés en la impartición de clases en las áreas de formación profesional de los alumnos, se debe pagar más al docente y por supuesto tener un alojamiento de la página que permita acceder sin problemas.

\section{Referencias}

Casañ Pitarch, R. (2019). Principios Pedagógicos en la adquisición de lenguas extranjeras mediante el enfoque basado en juegos digitales. Tonos Digital, 37(0).

Carrió Pastor, M. L., \& Romero Forteza, F. (2019). La planificación temporal y la evaluación en la formación online de futuros docentes en el AICL.

Fuentes, A. Y. P., \& Navas, M. F. (2019). Análisis Multidimensional del aprendizaje de lengua extranjera en ESPORTS. Revista Electrónica de Investigación y Docencia (REID), 69, 86.

Ortega Suarez, M. Á. (2019). Procesos cognitivos, metodologías comunicativas y uso de literatura en el aprendizaje de segundo idioma.

Ríos Márquez, S. A., \& González Martínez, H. D. (2019). Aprendizaje de vocabulario matemático a través del diseño de una unidad didáctica enmarcada en los principios del enfoque CLIL (Content and Language Integrated Learning).
Rodríguez, N. E. (2010). Competencia saber comunicarse [objeto de aprendizaje]. Disponible en el sitio Web: http://www.ruv.itesm.mx/convenio/tabasco/oas/ sc/homedoc.htm. Disponible en el repositorio abierto de la cátedra de investigación de innovación en tecnología y educación del Tecnológico de Monterrey en: http://catedra.ruv.itesm.mx//handle/987654321/ 99

Rodríguez, N.E. (2013). La gestión del conocimiento mediado por los REA: la experiencia en una Universidad Tecnológica Mexicana. Revista Electrónica de Tecnología Educativa.(43),a225. Disponible en https://doi.org/10.21556/edutec.2013.43.330 